\title{
ORIGINAL ARTICLE \\ Sex-determining chromosomes and sexual dimorphism: insights from genetic mapping of sex expression in a natural hybrid Fragaria $\times$ ananassa subsp. cuneifolia
}

\begin{abstract}
R Govindarajulu ${ }^{1}$, A Liston ${ }^{2}$ and T-L Ashman ${ }^{1}$
We studied the natural hybrid (Fragaria $\times$ ananassa subsp. cuneifolia) between two sexually dimorphic octoploid strawberry species (Fragaria virginiana and Fragaria chiloensis) to gain insight into the dynamics of sex chromosomes and the genesis of sexual dimorphism. Male sterility is dominant in both the parental species and thus will be inherited maternally, but the chromosome that houses the sex-determining region differs. Thus, we asked whether (1) the cytotypic composition of hybrid populations represents one or both maternal species, (2) the sex-determining chromosome of the hybrid reflects the location of male sterility within the maternal donor species and (3) crosses from the hybrid species show less sexual dimorphism than the parental species. We found that $F . \times$ ananassa subsp. cuneifolia populations consisted of both parental cytotypes but one predominated within each population. Genetic linkage mapping of two crosses showed dominance of male sterility similar to the parental species, however, the map location of male sterility reflected the maternal donor in one cross, but not the other. Moreover, female function mapped to a single region in the first cross, but to two regions in the second cross. Aside from components of female function (fruit set and seed set), other traits that have been found to be significantly sexually dimorphic in the pure species were either not dimorphic or were dimorphic in the opposite direction to the parental species. These results suggest that hybrids experience some disruption of dimorphism in secondary sexual traits, as well as novel location and number of quantitative trait locus (QTL) affecting sex function.
\end{abstract}

Heredity (2013) 110, 430-438; doi:10.1038/hdy.2012.96; published online 21 November 2012

Keywords: Fragaria; hybrid; sexual dimorphism; sex chromosome; male sterility

\section{INTRODUCTION}

Natural hybrids can form when two species with incomplete reproductive isolation come into secondary contact (Rieseberg and Carney, 1998). Once viewed as 'evolutionary noise' (Wagner, 1970), hybrid zones are now being viewed as natural laboratories for ecological and evolutionary studies in speciation and diversification (for example, Rieseberg and Wendel, 1993; Sweigart, 2009) as they provide insight into the prevailing direction of gene flow, gene introgression and adaptation (for example, Nolte et al., 2009; Wallace et al., 2011). It also has been noted that natural hybrid zones are an underexploited source of information on reproductive isolation and mating (reviewed in Rieseberg and Blackman, 2010), early sex chromosome evolution (Veltsos et al., 2008) and the genetics of sexual dimorphism (Coyne et al., 2008). Hybrid zones may be where new sterility alleles are expressed (reviewed in Rieseberg and Blackman, 2010) or old ones rearranged (for example, Petit et al., 2010). Such novelty could lead to the evolution and spread of new sex-determining chromosomes (Veltsos et al., 2008; Pannell and Pujol, 2009). Moreover, because sexual dimorphism (or sex limitation) that evolved separately in the two species can breakdown in the hybrids (Parker and Partridge, 1998), the pattern of dissolution or re-expression of male traits in females can provide information on the origin and type of genetic control underlying sexual dimorphism (Coyne et al., 2008).
Although there are a handful of well-studied plant hybrid zones that involve gender dimorphic (that is, dioecious (males and females), or gynodioecious (females and hermaphrodites)) parental species; for example, Buggs and Pannell, 2007; Minder et al., 2007; Lexer et al., 2010; Wallace et al., 2011), there are few where we also have explicit knowledge of the location of sex-determining genes in the parental species. In fact, we have very few studies that compare genetic maps of sex determination in hybrids with their parent species (but see, Paolucci et al., 2010; Macaya-Sanz et al., 2011), or the level of sexual dimorphism in the hybrid to that of its parental species. Yet, it is in these systems where we will be most readily able to address questions of novel locations of sex-determining genes and of the effect of hybridization on sexual dimorphism.

As a first step in addressing these gaps, we investigated the level of population admixture and the location of the sex-determining region in two populations of Fragaria $\times$ ananassa subsp. cuneifolia, a natural hybrid of two octoploid species, Fragaria chiloensis and Fragaria virginiana. These are the same two species that were cultivated in Europe in the 1700s, and hybridized to produce the cultivated strawberry F. $\times$ ananassa subsp. ananassa (Darrow, 1966).

Both parental species show gender dimorphism and sexual dimorphism in secondary traits (Ashman, 2003, 2005; Ashman et al., 2011; Spigler et al., 2011) but they differ in the chromosome

${ }^{1}$ Department of Biological Sciences, University of Pittsburgh, Pittsburgh, PA, USA and ${ }^{2}$ Department of Botany and Plant Pathology, Oregon State University, Corvallis, OR, USA Correspondence: Dr T-L Ashman, Department of Biological Sciences, University of Pittsburgh, Pittsburgh, PA 15260, USA.

E-mail: tia1@pitt.edu

Received 12 April 2012; revised 23 July 2012; accepted 27 August 2012; published online 21 November 2012 
that houses the sex-determining region (Goldberg et al., 2010). F. chiloensis is predominantly dioecious (Hancock and Bringhurst, 1979b) and recent mapping studies have revealed that sex is determined by a dominant sterility allele $(A)$ at the male function 'locus' and recessive sterility allele $(g)$ at the female function 'locus' and that these 'loci' colocalize on linkage group (LG) VI.A (Goldberg et al., 2010). F. virginiana is subdioecious, and sex expression is also controlled by a dominant male-sterility allele and a recessive femalesterility allele but here linkage between the two sex function 'loci' is less complete (that is, recombination can lead to hermaphrodites and neuters), and these major sex-determining loci are on LG VI.C (Spigler et al., 2010). The dominance and different chromosomal locations of male sterility means that, barring rearrangements, the location of sexdetermining genes in the hybrid should reflect that of the maternal donor species. That is, when F. chiloensis is the donor we expect male sterility to map to LG VI.A but when F. virginiana is the maternal donor it will map to VI.C. One can use species-specific plastome markers to identify the maternal donor of individuals and the populations they derive from (Minder et al., 2007; Arnold et al., 2010) to facilitate testing this hypothesis. In addition, as several of the sexually dimorphic secondary traits have been shown to be controlled, in part, by loci colocalizing with the sex-determining region (Ashman et al., 2011; Spigler et al., 2011), we predicted that dimorphism in these traits would be less in the hybrid than found in the pure species crosses.

Specifically, we sought to determine whether (1) two populations of $F$. $\times$ ananassa subsp. cuneifolia reflect a single parental species or a mixture of the two parental species as maternal donors; (2) the sexdetermining chromosome of the hybrid reflects the location of male sterility within the maternal donor species; and (3) crosses from the hybrid species show less sexual dimorphism than published reports for the parental species.

\section{MATERIALS AND METHODS}

\section{Species description and study populations}

F. $\times$ ananassa subsp. cuneifolia is a perennial stoloniferous herb that inhabits edges of pastures, pine forests, roadways and the interfaces of woods and back dunes (Hancock and Bringhurst, 1979a; Staudt, 1999). It is morphologically intermediate to the parental species (Salamone et al., in preparation; Staudt, 1999) and currently exists in a narrow hybrid zone that stretches from southern British Columbia to northern California (Staudt, 1999). Although the two progenitor species last shared a common ancestor 400 000-1 770000 years ago based on the $95 \%$ highest posterior density of a Bayesian dating analysis (Njuguna et al., 2012), the timing of secondary contact is unknown. Staudt (1989) speculated that hybridization occurred after glaciation in the Fraser River valley of British Columbia receded. Evidence from diagnostic plastome single-nucleotide polymorphisms indicates that either species can act as the maternal donor (Salamone et al., in preparation). Cytological and genetic evidence also suggests that both wild octoploid species and the cultivated hybrid $F . \times$ ananassa subsp. ananassa have a genomic structure of $\mathrm{AAA}^{\prime} \mathrm{A}^{\prime} \mathrm{BBB}^{\prime} \mathrm{B}^{\prime}$ (Bringhurst, 1990), and exhibit disomic inheritance $(2 n=8$ $x=56$ ) (Ashley et al., 2003; Lerceteau-Köhler et al., 2003; Rousseau-Gueutin et al., 2008). F. $\times$ ananassa subsp. cuneifolia is subdioecious with three sexual morphs (hermaphrodites, males and females) co-occurring within a population (Ashman personal observation; Staudt, 1999), similar to one of its progenitor species F. virginiana (Ashman and Hitchens, 2000).

We collected $32 \mathrm{~F} \times \times$ ananassa subsp. cuneifolia plants along transects through each of two populations in Benton County, OR, USA (Wren (WREN) $(44.5878 \mathrm{~N}, 123.4272 \mathrm{~W}, 133 \mathrm{~m})$ and Mary's Peak (MP) (44.5044 N, $123.55 \mathrm{~W}$, $1203 \mathrm{~m})$ ). All three sexual phenotypes were observed in these populations and females represented $\sim 50 \%$ in each population (Wren: $47 \%$, MP: $53 \%$ ). Plants were grown in $200 \mathrm{ml}$ pots filled with a 2:1 mixture of Fafard \#4 (Conrad Fafard) and sand in the greenhouse at the University of Pittsburgh. Plants received fertilizer and protection from pests as needed.

\section{Creation and cultivation of F. $\times$ ananassa subsp. cuneifolia} mapping populations

To map sex determination, we created two mapping populations by crossing plants derived from WREN and MP populations. In MP1 $\times$ WREN 2 cross a MP female (67\% fruit set) was pollinated with pollen from a WREN individual that was male fertile but set no fruit. In the WREN7 $\times$ MP10 cross, a WREN female with $96 \%$ fruit set was pollinated with pollen from a MP hermaphrodite (32\% fruit set). We pollinated female parents with pollen collected from the male parents during February through April 2010 and planted 144 seeds from each cross in May 2010. Seeds were planted in 72-well trays with a custom germination mix (Sunshine germination mix: Fafard \#4: sand), and exposed to 14 -h days and $15^{\circ} \mathrm{C} / 20^{\circ} \mathrm{C}$ night/day temperatures in a growth chamber. Germination was high (both crosses 95\%), and after 2 months of growth seedlings were transplanted into $200 \mathrm{ml}$ pots filled with a 2:1 mixture of Fafard \#4 and sand. At this time, we also produced two clones of each parent. All plants were exposed to $12{ }^{\circ} \mathrm{C} / 22^{\circ} \mathrm{C}$ night/day temperatures and 12 -h day light for 4 months before a 2-month dark treatment at $4^{\circ} \mathrm{C}$. Growth conditions during flowering were 11 -h day light at $12{ }^{\circ} \mathrm{C} / 18^{\circ} \mathrm{C}$ night/day temperatures. We hand-pollinated each flower on all plants three times per week with outcross pollen to ensure full potential fruit and seed set. During the entire course of study, all plants received seven beads of granular nutricote 13:13:13 N:P:K fertilizer (Chisso-Ashai fertilizer, Sun Gro Horticulture, Bellevue, WA, USA) and were protected from pests as needed.

\section{Sex expression and phenotype data}

Sex expression was scored on each plant at least twice during flowering. As in previous studies (Goldberg et al., 2010; Spigler et al., 2010), we scored male function qualitatively based on the presence or absence of pollen production and this was assessed in at least two flowers per plant. Individuals with yellow anthers visibly releasing pollen were scored as 'male-fertile', whereas plants that produced white vestigial stamens and whose anther sacs lacked pollen were scored as 'male-sterile'. Female function was quantitatively estimated as the percentage of flowers that produced fruit ('fruit set'). To be consistent with the previous qualitative mapping of female function in F. virginiana (Spigler et al., 2008) and F. chiloensis (Goldberg et al., 2010), we considered plants with $\geqslant 5 \%$ fruit set as 'female fertile' and those with $\leqslant 5 \%$ fruit set as 'female sterile'. We scored sex expression on all the flowering plants in the mapping populations and present the data for the representative subset that were genotyped. In addition, we scored several phenotypic traits that have been shown to be sexually dimorphic in F. virginiana and/or F. chiloensis (proportion seed set, anther number per flower, flowers per plant, leaf number per plant and runner number per plant) following the protocols described in Spigler et al. (2011).

For each F1 mapping population, we determined whether there was sexual dimorphism between male-sterile and male-fertile morphs using $t$-tests. To facilitate comparisons with published indices of sexual dimorphism in parental species (Ashman et al., 2011; Spigler et al., 2011), we calculated a sexual dimorphism index following McDaniel (2005) as $\mid\left(x_{\mathrm{MS}}-x_{\mathrm{MF})} \mid /[(\right.$ s.e.MS + s.e. MF $) / 2$ ], where $x$ and s.e. are the mean and standard error, respectively, for each trait for male-sterile (MS) and male-fertile (MF) morphs.

\section{DNA extraction}

DNA was extracted from $10-15 \mathrm{mg}$ of silica-dried young leaf tissue from the 32 plants per population for cytotyping and from progeny ( 85 from MP12 $\times$ WREN2, 90 from WREN7 $\times$ MP10) and two replicates of the parents of the two crosses for genetic mapping. We used a CTAB extraction protocol (Doyle and Doyle, 1987) modified to accommodate a 96-well high-throughput format. DNA was quantified using a Spectromax 190 spectrophotometer (Molecular Devices, Sunnyvale, CA, USA) and diluted to $0.3 \mathrm{ng}^{-1} \mathrm{l}^{-1}$ with deionized sterile water for PCR reactions.

\section{Cytotyping}

To distinguish the parental donor of $F . \times$ ananassa subsp. cuneifolia individuals, we screened each individual for two single-nucleotide polymorphisms that differentiate F. virginiana and F. chiloensis chloroplast genomes (Njuguna et al., 2012; Salamone et al., in preparation). Single-nucleotide polymorphisms 
within the intron of petD and an exon of $n d h \mathrm{~F}$ were screened in 32 plants per population using the dCAPs technique (Neff et al., 1998).

Specifically, we amplified the two chloroplast regions petD (primers petDintron-77875F (5'-GGATAGGCTGGTTCGTTTGA-3'), petDintron78409R (5'-GCTCGAGCATGAATCAACAG- $\left.3^{\prime}\right)$ ) and $n d h \mathrm{~F}$ (ndhF-113272F (5'-AAAATCCCCGACACGATTAG- ${ }^{\prime}$ ), ndhF-113799R (5'-ACCGTTCATTCCACTTCCAG- $\left.\left.3^{\prime}\right)\right)$. PCR reactions included $1 \times$ PCR buffer $(10 \times$ buffer with $\mathrm{MgCl}_{2}$, Qiagen, Valencia, CA, USA), $100 \mu \mathrm{m}$ of each dNTP, $0.5 \mu \mathrm{m}$ of each forward and reverse primer, 1.5 units of Taq polymerase and $1 \mu \mathrm{l}$ of genomic DNA in a $20 \mu \mathrm{l}$ reaction. PCR amplification began with a hot start of $95^{\circ} \mathrm{C}$ for $2 \mathrm{~min}$ to activate the Taq polymerase (New England Biolabs, Beverly, MA USA) followed by $94^{\circ} \mathrm{C}$ for a 45 -s denaturation step, followed by 35 cycles of: (1) 45 -s denaturation at $94{ }^{\circ} \mathrm{C}$; (2) 30 -s annealing at $51{ }^{\circ} \mathrm{C}$; (3) 60 -s extension at $72^{\circ} \mathrm{C}$ and a final extension for $8 \mathrm{~min}$ at $72^{\circ} \mathrm{C}$. The amplified PCR products were purified using Qiagen PCR purification kit. To validate the singlenucleotide polymorphisms for cytotyping, we sequenced purified products on ABI 3730XL DNA analyzer (Life Technologies, Carlsbad, CA, USA). For dCAPs, we digested $6 \mu \mathrm{l}$ of purified $n d h \mathrm{~F}$ product from each sample with five units of the restriction enzyme $M s l \mathrm{I}$ for $2 \mathrm{~h}$ at $37^{\circ} \mathrm{C}$, and $6 \mu \mathrm{l}$ of purified petD product with five units of $T a q^{\alpha} \mathrm{I}$ at $65^{\circ} \mathrm{C}$ for $2 \mathrm{~h}$. The recognition site of MslI includes the underlined variable site (CATTG^AAGTA/CATTGAAGTG) within $n d h \mathrm{~F}$ and that of $T a q^{\alpha} \mathrm{I}$ includes the underlined variable site $(\mathrm{T} \wedge \mathrm{CGA} / \mathrm{TCAA})$ in petD. Products were assayed on agarose gels and speciesspecific cytotypes identified as follows: (1) $n d h \mathrm{~F}$ locus, a 500-bp (uncut) product identifies $F$. virginiana whereas two fragments (336 and 166-bp product) corresponds to the F. chiloensis cytotype; (2) two pet $\mathrm{D}$ fragments (56 and $44 \mathrm{bp}$ ) differentiate the $F$. chiloensis cytotype from the $F$. virginiana (uncut) cytotype.

\section{Nuclear marker analysis and genotyping}

As we were interested in determining the location of sex-determining region and assessing the homology of the sex-determining chromosome in F. $\times$ ananassa subsp. cuneifolia to its progenitors, we genotyped the mapping populations using primer pairs that have been shown to amplify DNA markers (simple sequence repeats or genes) on LG within the homoeologous group (HG) that houses the sex-determining chromosomes in F. virginiana and $F$. chiloensis (that is, HG VI, Goldberg et al., 2010; Spigler et al., 2010). Thirtynine primer pairs were used to construct genetic map of HG VI and map the sex-determining region in $F . \times$ ananassa subsp. cuneifolia in the WREN7 $\times$ MP10 cross and 10 of these were used to construct genetic map for the MP12 $\times$ WREN2 cross.

For nuclear markers, PCR reactions were performed using the Poor Man's PCR protocol as previously described (Spigler et al., 2008; Goldberg et al., 2010). We multiplexed PCR products from 2 to 4 primers by mixing $1.3 \mu \mathrm{l}$ aliquots from each reaction with $0.2 \mu \mathrm{l}$ LIZ500 standard and $10.5 \mu \mathrm{l} \mathrm{Hi-Di}$ formamide (Applied Biosystems). Fragment analysis and genotyping were conducted using ABI 3730XL DNA analyzer and GeneMapper ver 3.0 (Applied Biosystems, Life Technologies, Carlsbad, CA, USA).

\section{Construction of genetic maps}

We used the single-dose restriction fragment marker analysis and a pseudo test-cross strategy to construct genetic linkage maps for $F . \times$ ananassa subsp. cuneifolia, as is customary in polyploids (Wu et al., 1992; Garcia et al., 2006) As a single-primer pair can amplify multiple PCR products as a result of paralogs in the octoploid genome, we considered each PCR product a singledose marker and scored it as present (1) and absent (0) in the progeny. Using $\chi^{2}$ tests, we evaluated each polymorphic marker for goodness of fit according to the expected Mendelian segregation ratio of either 1:1 if present in only one parent or 3:1 if present in both parents. In each cross, we discarded markers that deviated from the expected segregation ratios at $P \geqslant 0.0001$ and retained all other markers for mapping. We mapped the marker data from each primer pair in JoinMap 4.0 (Van Ooijen, 2006) to determine whether markers from a given primer pair represented co-segregating alleles at a single locus. The PCR products from single primer were considered to be allelic at a locus if they mapped in the same location and were in repulsion. For these, we retained one member of each pair to be consistent with the single-dose marker approach
(Wu et al., 1992; Spigler et al., 2010). For PCR products that were linked but did not map to the same location, we checked the raw data to reevaluate the genotypes and reconfirm scores as in past work (Spigler et al., 2010).

We constructed separate maps of HG VI for maternal and paternal parents by considering them as cross-pollinator population type (outbred full-sib family cross). Before mapping, we excluded markers or individuals that were missing data for $>25 \%$. This resulted in the exclusion of only two individuals from one cross $(\mathrm{MP} 12 \times$ WREN2). Initial LGs were inferred at logarithm of odds $($ LOD) $\geqslant 6$. Additional ungrouped markers were assigned at LOD $>4$ threshold using strongest cross-link values. Marker order and map distance were determined using the Kosambi mapping function with default parameter settings (minimum LOD threshold of 1.0, recombination threshold of 0.40 and jump threshold of 5.0) to include maximum number of markers to identify homoeologs within HG VI. We also applied strict mapping parameters (minimum LOD threshold of 3.0, recombination threshold of 0.35 and jump threshold of 3.0) to test stability of linkage between markers within each homoeolog and found no major differences from the less strict mapping parameter settings. We considered genetic maps derived from default mapping parameter for further analyses. Graphical maps were constructed in MapChart 2.1 (Voorrips, 2002).

The LGs within each parental HG VI map were assembled on the basis of strongest cross-link values of LOD $\geqslant 4$ between markers on the LGs or by comparing the LG with a putative homoeolog in the other parent (based on shared markers fitting 3:1 segregation ratio). LGs were named VI.A, VI.B, VI.C and VI.D based on the LG-specific markers on homoeologous LGs in F. virginiana and F. chiloensis genetic maps (Goldberg et al., 2010; Spigler et al., 2010). Mapped markers that deviated in expected segregation ratios at $0.0001<P<0.01$ were identified as skewed and are denoted along with the direction of skew (that is, under or over representation of the heterozygote).

\section{Qualitative and quantitative mapping of sex expression}

To qualitatively map sex function traits, we considered male sterility and female sterility as single-dose markers and tested for fit to Mendelian segregation ratios (1:1 and 3:1) as in past work (Spigler et al., 2008; Goldberg et al., 2010).

To quantitatively map female function, the mapping populations were treated as 'doubled haploid populations', which can evaluate quantitative trait locus (QTL) more efficiently (Van Ooijen, 2004), but require the exclusion of markers found in both parents that segregate 3:1 (that is, 'hk $\times$ hk' segregation types in JoinMap) from the genetic map (Van Ooijen, 2006). We conducted QTL analysis on each parent map separately using MapQTL5 (Van Ooijen, 2004). As in past work (Spigler et al., 2010, Ashman et al., 2011), we used Kruskal-Wallace analysis followed by interval mapping and composite interval mapping (multiple QTL model, 'MQM', in MapQTL) to identify potential single-marker associations and QTL for fruit set. Only the markers identified as significant in the MQM were also significant in the single-marker tests after Bonferroni correction, thus we only report the results of the more conservative MQM results. The QTLs (delineated by 2-LOD intervals) detected through MQM are depicted on the original LG map for simplicity using an autoqtl function in MapChart (Voorrips, 2002). Results were unchanged when QTL analysis was performed without the 'marker' male sterility (data not shown). We tested for epistatic effects between male sterility and female function QTL when they did not overlap, as well as between multiple female function QTL when present, following the analysis of variance approach of Spigler et al. (2011).

To assess macrosynteny of the sex-determining chromosomes in $F . \times$ ananassa subsp. cuneifolia with the parental species, we aligned the LGs carrying male sterility found here with those published for $F$. virginiana and F. chiloensis (Goldberg et al., 2010; Spigler et al., 2010). We also included the homoeolog from a cross between two diploid hermaphrodite species, Fragaria vesca and Fragaria nubicola ('Fv $\times$ Fn', Sargent et al., 2009) for reference.

\section{RESULTS Cytotyping}

Both F. $\times$ ananassa subsp. cuneifolia populations contained F. chiloensis and F. virginiana cytotypes, but they differed dramatically 
Table 1 Frequency of $F$. chiloensis and $F$. virginiana cytotypes found in two $F$. $\times$ ananassa subsp. cuneifolia populations

\begin{tabular}{|c|c|c|c|c|c|}
\hline \multirow[t]{2}{*}{ Population } & \multirow[t]{2}{*}{ Cytotype } & \multicolumn{2}{|c|}{ Gene } & \multicolumn{2}{|c|}{ Consensus } \\
\hline & & petD & $n d h F$ & Total & $\%$ \\
\hline \multirow[t]{2}{*}{ MP } & F. chiloensis & 29 & 29 & 29 & 93.5 \\
\hline & F. virginiana & 2 & 2 & 2 & 6.5 \\
\hline \multirow[t]{2}{*}{ WREN } & F. chiloensis & 2 & 2 & 2 & 6.6 \\
\hline & F. virginiana & 30 & 27 & 30 & 93.4 \\
\hline
\end{tabular}

Abbreviation: MP, Mary's Peak.

in the frequency of the two types (Table 1). The F. chiloensis cytotype was predominant in the MP population (93.5\%) whereas the F. virginiana one was at Wren (93.4\%). The parents of the mapping populations had the majority cytotypes of their respective populations.

\section{Genetic maps of HG-VI}

MP12 $\times$ WREN2. Ten primer pairs amplified 75 PCR products, 57 of which met our criteria for map construction. Five of the 25 markers (19 (1:1 markers) and 6 (3:1 markers)) used for the maternal map co-segregated so 20 markers were retained for mapping. In the paternal map, 12 of the 36 markers (30 (1:1 markers) and 6 (3:1 markers)) co-segregated, thus 24 were retained for mapping. From these, seven and three markers (maternal and paternal, respectively) were unlinked and not included in the final map.

We assembled the LGs of the maternal map (Supplementary Figure 1, top) into two homoeologous chromosomes (VI.A and VI.B) based on synteny with the progenitor species and the WREN map (see below). The size of LGs ranged from 20 to $66.2 \mathrm{cM}$, with 4 to 7 markers per group.

The paternal map (Supplementary Figure 1, bottom) was resolved into five LGs that were assembled into three homoeologous chromosomes (VI.A, VI.B and VI.C). The size of the LGs ranged from 1.2 to $41.1 \mathrm{cM}$, with 2 to 8 markers per group. In the paternal map only, $14 \%$ of the 21 mapped markers were skewed. There were three single markers (SCAR2, CFCVT017 and UFFxa01E03) that showed moderate segregation distortion $(P<0.01)$ and over-representation of heterozygous gametes among genotypes.

WREN7 $\times$ MP10. Thirty-nine primer pairs amplified 225 products. From these, 142 met our criteria for genetic map construction. In total, 13 of the 79 markers (54 (1:1 markers) and 25 (3:1 markers)) used for maternal map co-segregated, so 66 were retained for final mapping. In the paternal map, 16 of the 88 markers (63 (1:1 markers) and 25 (3:1 markers)) co-segregated and thus 72 were retained for mapping. In all, 25 and 30 markers of the final sets were unlinked (maternal and paternal, respectively), thus are not included in the final maps.

The maternal map of HG VI (Supplementary Figure 2, top) comprises 41 markers that resolved into 8 LGs. We assembled six of these LGs into four homoeologous chromosomes and identified LGs VI.A through VI.D based on the presence of LG-specific markers in comparison with the progenitor genetic maps. For instance, SCAR2 on VI.A, F.v.a108 on VI.C, F.v.B119 on VI.D in both F. virginiana and F. chiloensis maps (Goldberg et al., 2010; Spigler et al., 2010) and tandemly duplicated EMFv104 markers along with other markers as LG VI.B in F. chiloensis (Goldberg et al., 2010). These LGs ranged in size from 13.4 to $82.1 \mathrm{cM}$, with 3 to 17 markers per group. Two small
LGs (two markers) were not assigned to a homoeologous chromosome. Only one confirmed case of a duplicated marker was found (CFVCT006 on LG VI.A).

The paternal map of HG VI (Supplementary Figure 2, bottom) included 42 markers and resolved into 7 LGs, which we assembled into 4 LGs homologous to the maternal map. Three small LGs (two markers each) were not assigned to homoeologous chromosomes. LGs had 5 to 17 markers per group and ranged from 25.7 to $90 \mathrm{cM}$.

In this cross, 16 (19\%) of the mapped markers did not fit the expected Mendelian ratios. Of these, 69\% where paternal markers whereas $31 \%$ where maternal ones. These were most often single markers (Supplementary Figure 2, bottom). One potentially interesting case, however, is the two products of CFCVT017 on LG VI.B close to the QTL for fruit set in both maternal and paternal maps that showed a modest $(P<0.01)$ under-representation of heterozygous gametes among genotypes.

\section{Phenotypic sex expression: variation and mapping}

Phenotypic sex expression. Of the 85 genotyped progeny from MP1 $\times$ WREN2 cross, 40 progeny were male sterile and 45 were male fertile, thus male function segregated 1:1 (Figure $2 \mathrm{a} ; \chi^{2}=0.30$, $P=0.59)$. In this cross, female function also segregated $1: 1\left(\chi^{2}=0.42\right.$, $P=0.51)$ as 46 were female fertile and 39 were female sterile. The majority $(92 \%)$ of progeny were either female (male sterile and female fertile; $47 \%$ ), or male (male fertile and female sterile; $45 \%$ ), and six were hermaphrodites. Six females had fruit set lower than $75 \%$ (Figure 2a).

In the WREN7 $\times$ MP10 cross, 47 of the genotyped progeny were male sterile and 43 were male fertile, thus male function segregated $1: 1$ (Figure $2 b ; \chi^{2}=0.17, P=0.67$ ) in this cross as well. When female function was scored qualitatively, $92 \%$ of progeny were female fertile and female fertility deviated significantly from both 1:1 and 3:1 (both $P<0.0001$ ), precluding qualitative mapping (see below). Overall, 36 (40\%) progeny were hermaphrodite, 47 (52\%) female and $7(0.07 \%)$ male (Figure $2 \mathrm{~b}$ ).

Male function mapping. The results from both the crosses confirm the dominance of male sterility over male fertility, and in both map crosses male sterility mapped to one of the homoeologs within HG VI (Figure 1; Supplementary Figures 1 and 2). In MP12 $\times$ WREN2, male sterility mapped to the bottom of VI.A with tight linkage to two markers, PSContig6115 (LOD 10.0) and EMFn153 (LOD 5.1). In WREN7 $\times$ MP10, male sterility was linked in coupling to EMFv104_143 at a very high LOD (21.4). This marker is one of three tandemly duplicated products of the EMFv104 primer pair on LG VI.B (for example, EMFv104_143, EMFv104_135 and EMFv104_133).

Female function mapping. Qualitative mapping of female function in MP12 $\times$ WREN2 indicated that female function was linked to male sterility (Figure 1a). Similar to that seen in F. chiloensis (Goldberg et al., 2010), female fertility was dominant to female sterility and mapped in coupling with male sterility in the maternal parent. The QTL analysis of proportion fruit set is consistent with the qualitative mapping in this cross. A major QTL for proportion fruit set was found on LG VI.A in maternal map with a LOD score of 46.8 in MQM (Supplementary Figure 1). This QTL explained 93.2\% of the variation in fruit set and its peak was within $1.27 \mathrm{cM}$ of male sterility.

In the WREN7 $\times$ MP10 cross, only a quantitative approach to mapping female function was possible. In the maternal map, a QTL that explained $91.3 \%$ variation in fruit-setting ability colocalized with male sterility on LG VI.B with a LOD score of 47.2 (Supplementary 


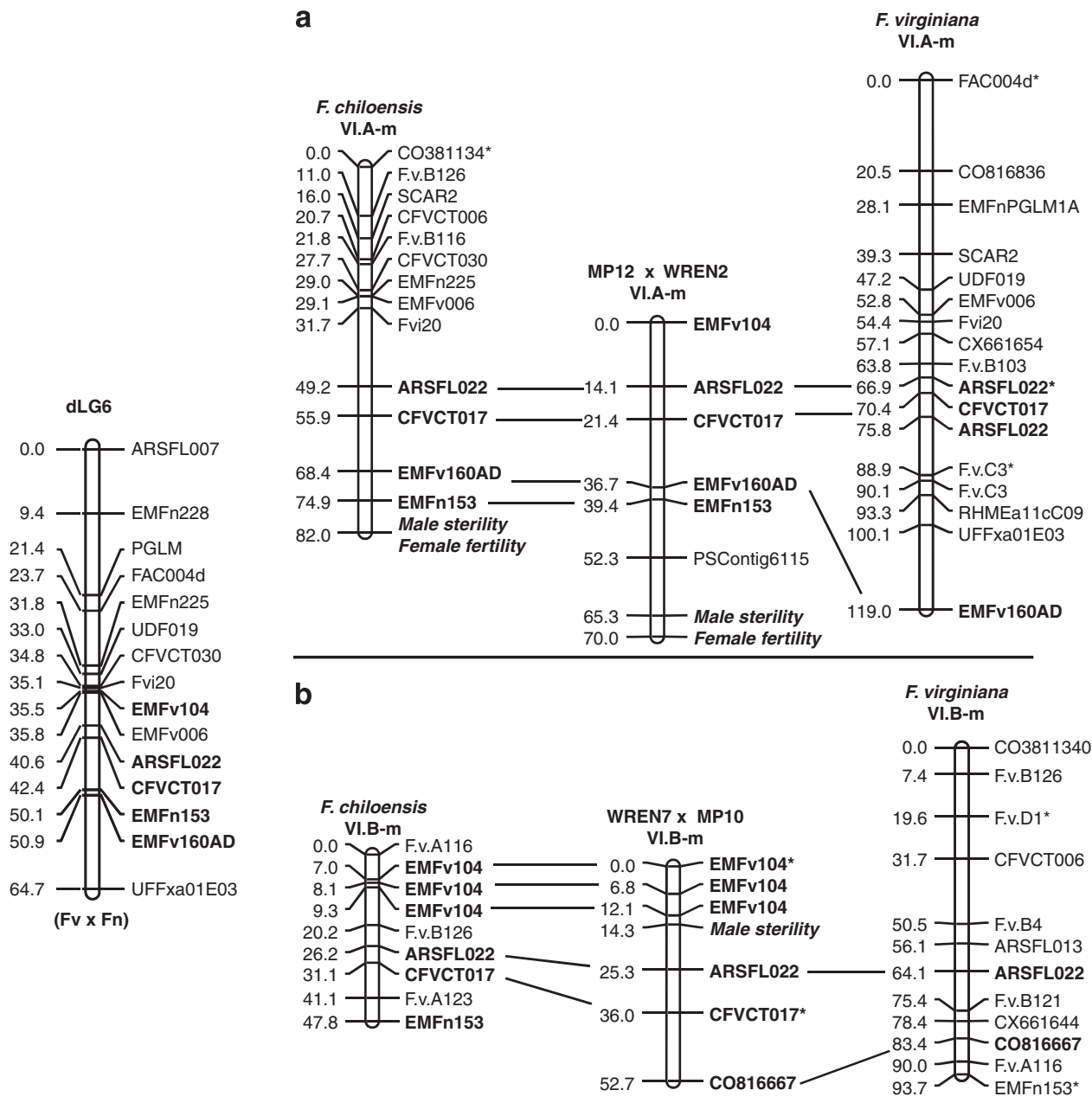

Figure 1 Comparison of male sterility carrying sex-determining chromosomes from both cross $(\mathbf{a}, \mathbf{b})$ to the corresponding homoeologs of $F$. virginiana (Spigler et al., 2010) and F. chiloensis (Goldberg et al., 2010) and the homoeologous LG6 in hermaphroditic diploid cross (Fv $\times$ Fn, adapted from Sargent et al., 2009) from previously published maps. Simple sequence repeats on the corresponding homoeologs in octoploids are connected by lines and are highlighted in bold to indicate synteny with the diploid reference homoeologous LG (dLG6). Phenotypic trait marker representing the putative sex determining loci (male sterility/female fertility) are indicated in bold and italicized. Markers denoted by asterisk had skewed segregation ratios $(0.0001<P<0.01)$.

Figure 2). In the paternal map, a QTL explaining 10.6\% variation for fruit set was linked to CFVCT017 with LOD score of 1.89 and this QTL had a negative additive effect on fruit set. However, no significant epistatic interaction was found between this QTL and the female function QTL in the maternal parent when tested using analysis of variance $(P>0.05)$.

\section{Macrosynteny of sex-determining chromosomes}

We compared the linkage map of the male sterility-determining chromosomes with their homoeologs in F. virginiana and F. chiloensis and in hermaphrodite diploid cross (dLG 6) (Figure 1). This revealed macrosynteny and only small differences in colinearity among these LGs. For instance, although the linkage map of the sex-determining chromosomes from both $F . \times$ ananassa subsp. cuneifolia crosses share three to five markers with LG 6 in diploid Fv $\times$ Fn cross, the order of EMFv160AD and EMFn153 in the MP12 $\times$ WREN2 cross agrees with the order in F. chiloensis but not $\mathrm{Fv} \times \mathrm{Fn}$ (Figure 1a). The synteny with $F$. chiloensis is in accord with fact that the maternal parent (MP12) has the F. chiloensis cytotype. A notable difference, however, is the greater estimated distance $(30 \mathrm{vs} 7 \mathrm{~cm}$ ) between the marker EMFn153 and male sterility in the F. $\times$ ananassa subsp. cuneifolia cross than in the F. chiloensis cross (Goldberg et al., 2010). In WREN7 $\times$ MP10, male sterility mapped above the three markers (ARSFL022, CFVCT017 and EMFn153) that subtended male sterility in MP12 $\times$ WREN2 and very close to a set of tandemly duplicated EMFv104 markers on LG VI.B. This location is not in agreement with the expected location of male sterility (on the tip of LG VI.C) given the F. virginiana cytotype of the maternal parent (WREN7). The tandemly duplicated set of EMFv104 markers, however, is similar to their arrangement on LG VI.B in F. chiloensis (Figure 1b; Goldberg et al., 2010) an alignment not observed in F. virginiana (Figure 1b; Spigler et al., 2010). Two markers located below male sterility (ARSFL022, CFVCT017) are shared by LG VI.B in F. chiloensis and two (ARSFL022, CO816667) by LG VI.B in F. virginiana (Figure 1b).

\section{Sexual dimorphism}

Proportion fruit set and proportion seed set were strongly sexually dimorphic in the progeny of both $F . \times$ ananassa subsp. cuneifolia crosses while ovule number was dimorphic in one cross (Table 2). In contrast, sexual dimorphism was nonexistent in anther number, runner number and leaf number, and was only weakly $(P<0.08)$ dimorphic for flower number in the WREN7 $\times$ MP10 cross (Table 2). 
The direction of dimorphism for flower number (male-sterile $>$ malefertile morphs) was opposite of that published for either of the pure species crosses (male-sterile $<$ male-fertile morphs; Ashman et al., 2011; Spigler et al., 2011), as was the direction of dimorphism for ovule number in the WREN7 $\times$ MP10 cross (male-sterile $<$ malefertile morphs; Ashman and Hitchens, 2000; Spigler et al., 2011).

\section{DISCUSSION}

Cytotypic composition of $F$. $\times$ ananassa subsp. cuneifolia hybrid populations

Our results show for the first time that natural hybrid populations of F. $\times$ ananassa subsp. cuneifolia contain cytotypes from both maternal
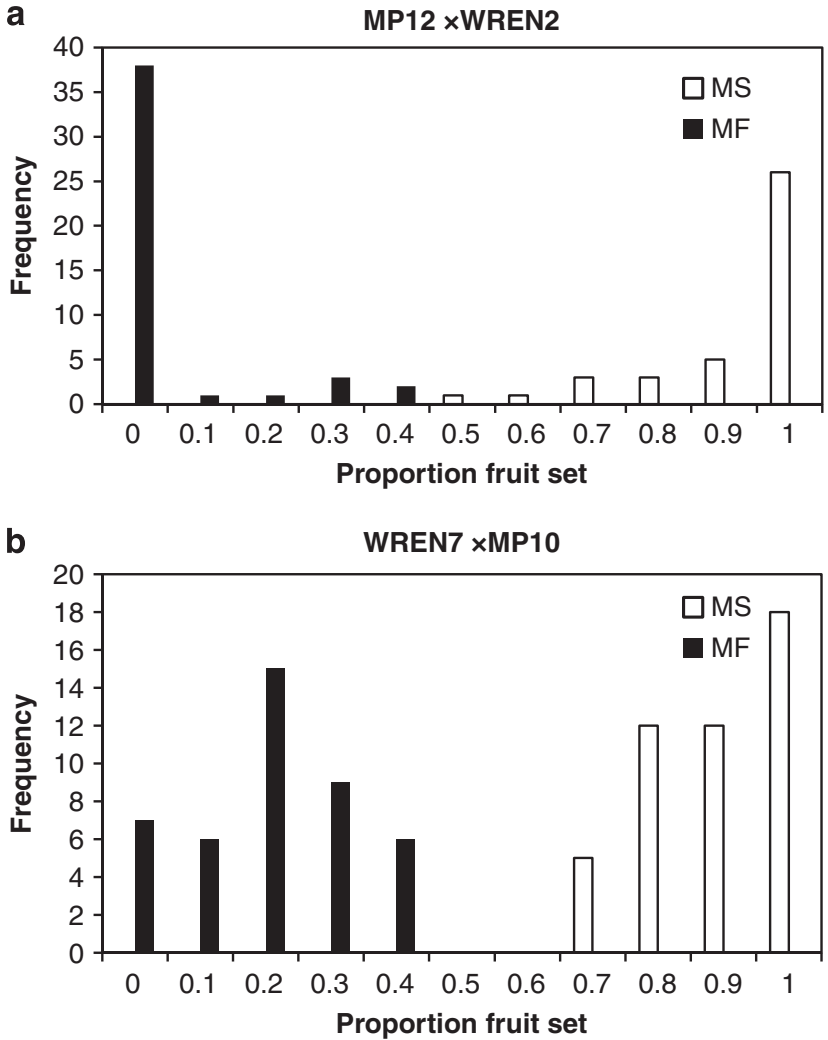

Figure 2 Frequency histograms of proportion fruit set (female function) for male sterile (MS) and male fertile (MF) progeny of $F . \times$ ananassa subsp. cuneifolia crosses. (a) MP12 $\times$ WREN2. (b) WREN7 $\times$ MP10 cross. species but that individual populations show a strong bias toward one cytotype or the other. The presence of both cytotypes suggests that both species contributed as maternal parents to the studied populations, and that admixture is still occurring, or that there was paternal leakage, although the latter is very rare in flowering plants (for example, McCauley et al., 2007) and was not detected in our crosses (unpublished data). Extreme bias in cytotypes is not uncommon in hybrid species, and can reflect the signature of the historical range of the dominant maternal species or of selective forces that favor one cytotype (Minder et al., 2007; Arnold et al., 2010). The two populations here are $13 \mathrm{~km}$ apart and are both near the margin of F. virginiana's range, although the MP population is closer to the coast. On the other hand, the MP population is on the highest peak $(1249 \mathrm{~m})$ in the Oregon Coast Range, an unusual habitat for F. chiloensis and thus could reflect a remnant population, or a long distance migrant, which was then subject to gene flow from $F$. virginiana. Broad sampling is underway to determine whether there is a geographic or edaphic pattern to the dominance of maternal cytotype and to assess the extent and direction of admixture in the nuclear genome across the hybrid zone. Such work will provide a landscape assessment of introgressive hybridization between $F$. chiloensis and F. virginiana, as well as indicate the potential for spread of new sterility alleles across the hybrid zone to contribute to turnover in sex-determining chromosomes in the two parental octoploid species (Veltsos et al., 2008). Reciprocal transplant and selection analyses will also provide powerful means to assess fertility selection on male and female function (for example, Spigler and Ashman, 2011) in the context of the sex expression variation provided by the hybrid zone (see below). Variation in sex determination regions of $F . \times$ ananassa subsp.
cuneifolia crosses

Although there was variation in the location of sex function loci, major QTL were found in HG VI, confirming the important role of this HG in sex determination in the octoploid Fragaria species. A similar consistent involvement of a specific LG has been seen among Populus species, that is, all sex-containing Populus linkage maps show sex determination on LG XIX, although in different positions and sometimes segregating in different parental genders (Pakull et al., 2009).

F. $\times$ ananassa subsp. cuneifolia is similar to both its parental species in that male sterility mapped in the female parent and was dominant to male fertility in both crosses. In one cross $($ MP12 $\times$ WREN2), male sterility mapped to the location predicted by maternal cytotype

Table 2 Trait means $( \pm$ s.e.) for MS and MF F1 progeny in the $\boldsymbol{F} . \times$ ananassa subsp. cuneifolia map crosses and SD indices

\begin{tabular}{|c|c|c|c|c|c|c|c|c|}
\hline \multirow[t]{2}{*}{ Trait } & \multicolumn{4}{|c|}{ MP12 × WREN2 } & \multirow[b]{2}{*}{ MS } & \multicolumn{3}{|c|}{ WREN7 $\times M P 10$} \\
\hline & $M S$ & $M F$ & $\mathrm{P}^{\mathrm{a}}$ & $S D$ index ${ }^{b}$ & & $M F$ & $\mathrm{~Pa}^{\mathrm{a}}$ & $S D$ inde $x^{b}$ \\
\hline Proportion fruit set & $0.89(0.20)$ & $0.04(0.11)$ & $<0.0001$ & 35.81 & $0.84(0.11)$ & $0.16(0.11)$ & $<0.0001$ & 43.25 \\
\hline Proportion seed set & $0.74(0.25)$ & $0.13(0.30)$ & $<0.0001$ & 14.59 & $0.88(0.10)$ & $0.64(0.34)$ & $<0.0001$ & 7.43 \\
\hline Flower number & $7.0(3.6)$ & $8.2(3.8)$ & 0.16 & 2.02 & $16.0(5.3)$ & $14.0(5.2)$ & 0.08 & 2.55 \\
\hline Ovule number & $52.0(11.9)$ & $50.4(15.0)$ & 0.58 & 0.79 & $55.0(11.6)$ & 64.7 (14.2) & $<0.005$ & 5.06 \\
\hline Anther number & $20.7(4.1)$ & $20.2(3.7)$ & 0.53 & 0.89 & $20.2(1.8)$ & $20.2(1.3)$ & 0.96 & 0.08 \\
\hline Leaf number & $10.8(4.4)$ & $11.7(4.6)$ & 0.35 & 1.33 & $18.0(7.1)$ & $18.5(7.7)$ & 0.75 & 0.46 \\
\hline Runner number & $6.3(2.1)$ & $6.4(2.2)$ & 0.72 & 0.51 & $5.0(2.2)$ & $5.1(2.3)$ & 0.88 & 0.21 \\
\hline
\end{tabular}

Abbreviations: MF, male-fertile; MP, Mary's Peak: MS, male-sterile. SD sexual dimorphism.

aprogeny means were evaluated using $t$-test and $P<0.05$ indicated in bold.

${ }^{b} \mathrm{SD}$ index $=\mid\left(x_{\mathrm{MS}}-x_{\mathrm{MF}}\right) / /[(\mathrm{s} . \mathrm{e} \cdot \mathrm{MS}+\mathrm{s} . \mathrm{e} \cdot \mathrm{MF}) / 2]$, where $x$ and s.e. are the mean and standard error, respectively, for each trait MS and MF progeny. 
(F. chiloensis), but in the other cross (WREN7 $\times$ MP10) male sterility mapped to a novel location. The novel location could be the result of transposition during hybrid formation as such rearrangements can be common in some hybrids (for example, Lai et al., 2005). Alternately, it is possible that male sterility has a different location in the western subspecies of $F$. virginiana subsp. platypetala than in the eastern F. virginiana subsp. virginiana. Current hypotheses for the biogeographic history of $F$. virginiana suggest that it, like many species in North America, may have been separated into two vicarious groups as the result of uplifting of the Rocky Mountains (Staudt, 1999). Using morphological and RAPD data, Harrison et al. (1997) concluded that F. virginiana subsp. platypetala was substantially differentiated from the rest of F. virginiana, and was more closely related to $F$. chiloensis than to F. virginiana subsp. virginiana. Staudt (1999) also suggested that $F$. virginiana subsp. platypetala may itself been derived from recent hybrids of $F$. virginiana and $F$. chiloensis. Although there is little consensus regarding these designations (Hancock et al., 2004), data to date do fuel speculation that gene flow between F. virginiana and F. chiloensis could be responsible for the dynamic nature of the sexdetermining region. Future work mapping sex determination within F. virginiana subsp. platypetala and work underway characterizing the extent of admixture in the nuclear genome across the hybrid zone will help resolve these issues.

Female function was found to map to a single region (and female sterility was recessive to female fertility) in one cross but mapped to two regions in the other cross. A single major QTL for female function in MP12 $\times$ WREN2 was linked to male function on VI.A, consistent with the location and linkage phase in the genetic map of F. chiloensis (male sterility in coupling with female fertility; Goldberg et al., 2010), but the linkage between the two was not as tight and recombinants were formed. In this cross, we can infer the genotypes of the parents as $A G \mid a g$ for the maternal parent and $a g \mid a g$ for the paternal parent, creating a majority of non-recombinant male and female progeny, and a smaller fraction of recombinant progeny, that is, the low fruit-setting females and hermaphrodites (Figure 2a; Supplementary Table 1a). This also conforms to Charlesworth and Charlesworth's (1978) two-locus model for sex determination (also see Spigler et al. 2008).

Not only was the location of male sterility in WREN7 $\times$ MP10 not as predicted, but the finding of two QTL for female sterility was unexpected. Multiple QTL affecting female function could indicate that (1) rearrangements of loci in the hybrid has led to novel placements of existing loci or (2) the hybrid has unique femalesterility alleles. We discuss the present results in the context of these two possibilities.

The QTL for female function linked to EMFv104 on VI.B clearly represents a new location relative to published maps of parental species and coincides with the QTL for male sterility, a location that could reflect either a transposition or new sterility genes as the result of hybridization, or as mentioned above a difference in the location of the sex-determining genes derived from the western subspecies of F. virginiana. If transposition occurred, it may have involved linked genes or a single gene that has pleiotropic effect on both male and female function. Determining this would require finer mapping and a larger population size, as there is no clear evidence in our current map for any other transposed markers in this region.

The QTL for fruit set overlapping CVCT017 (and EMFn153) on LG VI.B in the paternal parent, could also represent a transposition of a QTL, possibly from VI.A, as the paternal parent has F. chiloensis cytotype, or given the allopolyploid origin (for example, $\mathrm{AA} \mathrm{A}^{\prime} \mathrm{A}^{\prime} \mathrm{BB}$ $\mathrm{B}^{\prime} \mathrm{B}^{\prime}$ ) of the parental species could represent an orthologous QTL affecting fruit set on VI.B, that is, from a different genome donor, or a novel sterility locus. An orthologous QTL is a viable hypothesis because a recent study of the cultivated hybrid strawberry (F. $\times$ ananassa subsp. ananassa) concluded that $\sim 25 \%$ of QTL for fruit traits were at orthologous positions on a different homoeologous LGs, that is, were putative 'homoeo-QTL' (Lerceteau-Köhler et al., 2012), and these may be segregating in the natural hybrid species as well. Moreover, there is no evidence for transposition of other markers, which might be expected if nonhomologous recombination triggered the QTL at this position. In fact, there was only one confirmed case of novel duplication (CFVCT006 on LG VI.A) in our map of the natural hybrid.

However, sterility can directly result from hybridization (reviewed in Maheshwari and Barbash, 2011). Of the many possible mechanisms, two have some support in plants: (1) negative genetic interactions between two or more loci fixed in the two parental species (for example, Dobzhansky - Muller incompatibilities; Fishman and Willis, 2001; Moyle and Nakazato, 2010; Maheshwari and Barbash, 2011), and (2) rearrangements (for example, pollen sterility QTL were located near rearrangement breakpoints in artificial Helianthus hybrids; Lai et al., 2005). Both are possibilities here because the characteristics of sex chromosomes, in particular, are thought to make them 'hotspots' for speciation genes (Qvarnstrom and Bailey, 2008), and rearrangements have been found in the octoploid species that involve LGs in HG VI (Spigler et al., 2010; Sargent et al., 2012). The negative additive effect of the fruit set QTL in paternal parent (reduces fruit set by 10\%) and the skewed segregation of the nearest marker (Supplementary Figure 1) might indicate the involvement of a Dobzhansky - Muller incompatibility. Further crosses are required to resolve whether female sterility at this location is the manifestation of different genes related to speciation in this region.

In the context of past models (Spigler et al., 2008; Goldberg et al., 2010), the diversity of sexual phenotypes in the WREN (Figure 2), including low fruiting females and moderately fruiting hermaphrodites, can be accounted for by recombination between the two female function QTL on LG VI.B (Supplementary Table 1B).

\section{Sexual dimorphism in hybrid $F$. $\times$ ananassa subsp. cuneifolia}

Aside from components of female function (fruit set and seed set), other traits that have been found to be significantly sexually dimorphic in the pure species were either not dimorphic in the hybrid crosses (anther number, runner number and leaf number, flower number; Staudt, 1999; Ashman et al., 2011; Spigler et al., 2011) or if they were dimorphic the direction of dimorphism was in the opposite to what has been observed in the pure species (ovule number and flower number in the WREN7 $\times$ MP10 cross; Table 2). These results suggest that hybrids experience some disruption of dimorphism in secondary sexual traits. Loss of dimorphism in hybrids has been interpreted as reflecting breakdown of modifiers or regulatory elements that are responsible for dimorphism and suggests that these traits were originally expressed in both sexes but that modifier evolved afterward (Coyne et al., 2008). This interpretation may also apply to the traits studied here because all are expressed in both sexes to some degree and modifiers may be linked to sex-determining region (Spigler et al., 2011). In one of the few studies to assess dimorphism after hybridization, Zluvova et al. (2005) found females from crosses between dioecious Silene latifolia and hermaphrodite Silene viscosa had anthers that developed beyond the stage characteristic for S. latifolia females. Thus, the hybrid had less dimorphism in anther size than the pure species, and they interpreted this as evidence that the suppression of anthers was brought about by a recessive allele that 
could be rescued by the genome of $S$. viscosa. However, they did not find the same effect for fruit set in the males. Our work is far from conclusive, but does suggest that studies of the effects of hybridization on sexual dimorphism in plants will be useful for gaining insight into the genetic underpinnings and evolutionary processes (Coyne et al., 2008) and will be exemplary complements to studies of early sex chromosome evolution in plants.

\section{CONCLUSION}

The work presented here suggests that hybrid zones of dioecious/ subdioecious plants are valuable and underutilized resource for studying the ecology and evolution of sex chromosomes and sexual dimorphism. Ecological work will be especially valuable to test hypotheses for the spread of new chromosomal sex determination systems across hybrid populations (Veltsos et al., 2008). In addition, surveys of sexual dimorphism in natural hybrid zones, along with the creation of experimental hybrids will provide novel insight into the evolution and control of sexual dimorphism during the evolution of sex chromosomes.

\section{DATA ARCHIVING}

Sequence data have been submitted to GenBank: JX064433JX064440; JX064449-JX064456. Genotype and phenotype data are deposited with Dryad: doi:10.5061/dryad.cn66t. Data files: Cuneifolia_data_files_readme_files.

\section{CONFLICT OF INTEREST}

The authors declare no conflict of interest.

\section{ACKNOWLEDGEMENTS}

We thank R Dalton, D Jackson, B McTeague, M Parks, T Sanfilippo and E York for assistance in the greenhouse, field and laboratory, anonymous reviewers for comments on the manuscript and the Ashman lab members for discussion. This work was supported by the National Science Foundation (DEB 1020523) to TLA and AL (DEB 1020271).

Arnold M, Tang S, Knapp S, Martin N (2010). Asymmetric introgressive hybridization among Louisiana Iris species. Genes 1: 9-22.

Ashley MV, Wilk JA, Styan SMN, Craft KJ, Jones KL, Feldheim KA et al. (2003). High variability and disomic segregation of microsatellites in the octoploid Fragaria virginiana Mill. (Rosaceae). Theor App/ Genet 107: 1201-1207.

Ashman T-L (2003). Constraints on the evolution of males and sexual dimorphism: field estimates of genetic architecture of reproductive traits in three populations of gynodioecious Fragaria virginiana. Evolution 57: 2012-2025.

Ashman T-L (2005). The limits on sexual dimorphism in vegetative traits in a gynodioecious plant. Am Nat 166: S5-S16.

Ashman T-L, Hitchens MS (2000). Dissecting the causes of variation in intra-inflorescence allocation in a sexually polymorphic species, Fragaria virginiana (Rosaceae). Am J Bot 87: 197-204.

Ashman T-L, Spigler RB, Goldberg M, Govindarajulu R (2011). Fragaria: a polyploid lineage for understanding sex chromosome evolution. In: Navajas-Pérez R (ed) New Insights on Plant Sex Chromosomes. Nova Science Publisher: Hauppauge, New York.

Bringhurst RS (1990). Cytogenetics and evolution in American. Fragaria. Hortscience 25: 879-881.

Buggs RJA, Pannell JR (2007). Ecological differentiation and diploid superiority across a moving ploidy contact zone. Evolution 61: 125-140.

Charlesworth B, Charlesworth D (1978). A model for the evolution of dioecy and gynodioecy. American Naturalist 112: 975-997.

Coyne JA, Kay EH, Pruett-Jones S, True J (2008). The genetic basis of sexual dimorphism in birds. Evolution 62: 214-219.

Darrow GM (1966). The strawberry: History, Breeding and Physiology. Holt, Rinehart and Winston: New York

Doyle JJ, Doyle JL (1987). A rapid DNA isolation procedure for small quantities of fresh leaf tissue. Phytochem Bull 19: 11-15.

Fishman L, Willis JH (2001). Evidence for Dobzhansky-Muller incompatibilites contributing to the sterility of hybrids between Mimulus guttatus and M. nasutus. Evolution 55 1932-1942
Garcia AAF, Kido EA, Meza AN, Souza HMB, Pinto LR, Pastina MM et al. (2006). Development of an integrated genetic map of a sugarcane (Saccharum spp.) commercial cross, based on a maximum-likelihood approach for estimation of linkage and linkage phases. Theor App/ Genet 112: 298-314.

Goldberg MT, Spigler RB, Ashman T-L (2010). Comparative genetic mapping points to different sex chromosomes in sibling species of wild strawberry (Fragaria). Genetics 186: 1425-1433.

Hancock JF, Bringhurst RS (1979a). Ecological differentiation in perennial, octoploid species of Fragaria. Am J Bot 66: 367-375.

Hancock JF, Bringhurst RS (1979b). Hermaphroditism in predominately dioecious populations of Fragaria chiloensis (L) Duchn. Bull Torrey Bot Club 106: 229-231.

Hancock JF, Serce S, Portman CM, Callow PW, Luby JJ (2004). Taxonomic variation among North and South American subspecies of Fragaria virginiana Miller and Fragaria chiloensis (L.) Miller. Can J Bot 82: 1632-1644.

Harrison RE, Luby JJ, Furnier GR, Hancock JF (1997). Morphological and molecular variation among populations of octoploid Fragaria virginiana and $F$. chiloensis (Rosaceae) from North America. Am J Bot 84: 612-620.

Lai Z, Nakazato T, Salmaso M, Burke JM, Tang S, Knapp SJ et al. (2005). Extensive chromosomal repatterning and the evolution of sterility barriers in hybrid sunflower species. Genetics 171: 291-303.

Lerceteau-Köhler E, Guérin G, Laigret F, Denoyes-Rothan B (2003). Characterization of mixed disomic and polysomic inheritance in the octoploid strawberry (Fragariax ananassa) using AFLP mapping. Theor Appl Genet 107: 619-628.

Lerceteau-Köhler E, Moing A, Guérin G, Renaud C, Petit A, Rothan C et al. (2012). Genetic dissection of fruit quality traits in the octoploid cultivated strawberry highlights the role of homoeo-QTL in their control. Theor App/ Genet 124: 1-19.

Lexer C, Joseph JA, van Loo M, Barbará T, Heinze B, Bartha D et al. (2010). Genomic admixture analysis in European Populus spp. reveals unexpected patterns of reproductive isolation and mating. Genetics 186: 699-712.

Macaya-Sanz D, Suter L, Joseph J, Barbara T, Alba N, Gonzalez-Martinez SC et al. (2011). Genetic analysis of post-mating reproductive barriers in hybridizing European Populus species. Heredity 107: 478-486.

Maheshwari S, Barbash DA (2011). The genetics of hybrid incompatibilities. Annu Rev Genet 45: 331-355.

McCauley DE, Sundby AK, Bailey MF, Welch ME (2007). Inheritance of chloroplast DNA is not strictly maternal in Silene vulgaris (Caryophyllaceae); Evidence from experimental crosses and natural populations. Am J Bot 94: 1333-1337.

McDaniel SF (2005). Genetic correlations do not constrain the evolution of sexual dimorphism in the moss Ceratodon purpureus. Evolution 59: 2353-2361.

Minder AM, Rothenbuehler C, Widmer A (2007). Genetic structure of hybrid zones between Silene latifolia and Silene dioica (Caryophyllaceae): evidence for introgressive hybridization. Mol Ecol 16: 2504-2516.

Moyle LC, Nakazato T (2010). Hybrid incompatibility 'Snowballs' between Solanum species. Science 329: 1521-1523.

Neff MM, Neff JD, Chory J, Pepper AE (1998). dCAPS, a simple technique for the genetic analysis of single nucleotide polymorphisms: experimental applications in Arabidopsis thaliana genetics. Plant J 14: 387-392.

Njuguna W, Liston A, Cronn R, Ashman T-L, Bassil NV (2012). Insights into phylogeny, sex function and age of Fragaria based on whole chloroplast genome sequencing. Mol Phylogenet Evol S1055-7903: 00352-1.

Nolte AW, Gompert Z, Buerkle CA (2009). Variable patterns of introgression in two sculpin hybrid zones suggest that genomic isolation differs among populations. $\mathrm{Mol} E \mathrm{Col} 18$ : 2615-2627.

Pakull B, Groppe K, Meyer M, Markussen T, Fladung M (2009). Genetic linkage mapping in aspen (Populus tremula L. and Populus tremuloides Michx.). Tree Genet Genomes 5: 505-515.

Pannell JR, Pujol B (2009). The paradoxical spread of a new Y chromosome a novel explanation. Trends Ecol Evol 24: 59-63.

Paolucci I, Gaudet M, Jorge V, Beritognolo I, Terzoli S, Kuzminsky E et al. (2010). Genetic linkage maps of Populus alba L. and comparative mapping analysis of sex determination across Populus species. Tree Genet Genomes 6: 863-875.

Parker GA, Partridge L (1998). Sexual conflict and speciation. Proc Royal Soc B Biol Sci 353: 261-274

Petit M, Guidat C, Daniel J, Denis E, Montoriol E, Bui QT et al. (2010). Mobilization of retrotransposons in synthetic allotetraploid tobacco. New Phytol 186: 135-147.

Qvarnstrom A, Bailey RI (2008). Speciation through evolution of sex-linked genes. Heredity 102: 4-15.

Rieseberg LH, Blackman BK (2010). Speciation genes in plants. Ann Bot 106: 439-455. Rieseberg LH, Carney SE (1998). Plant hybridization. New Phytol 140: 599-624.

Rieseberg LH, Wendel J (1993). Introgression and its consequences in plants. In: Harrison R (ed.) Hybrid Zones and the Evolutionary Process. Oxford University Press: New York, pp 70-114.

Rousseau-Gueutin M, Lerceteau-Kohler E, Barrot L, Sargent DJ, Monfort A, Simpson D et al. (2008). Comparative genetic mapping between octoploid and diploid Fragaria species reveals a high level of colinearity between their genomes and the essentially disomic behavior of the cultivated octoploid strawberry. Genetics 179: 2045-2060.

Salamone I, Govindarajulu R, Falk S, Parks M, Liston A, Ashman T-L (2012). Ecological and phenotypic intermediacy and some geographic pattern to genetic admixture in natural strawberry hybrid. (in preparation).

Sargent D, Passey T, Surbanovski N, Lopez Girona E, Kuchta P, Davik J et al. (2012). A microsatellite linkage map for the cultivated strawberry (Fragaria $\mathrm{x}$ ananassa) suggests extensive regions of homozygosity in the genome that may have resulted from breeding and selection. Theor App/ Genet 124: 1229-1240. 
Sargent DJ, Fernandez-Fernandez F, Ruiz-Roja JJ, Sutherland BG, Passey A, Whitehouse $A B$ et al. (2009). A genetic linkage map of the cultivated strawberry (Fragaria $x$ ananassa) and its comparison to the diploid Fragaria reference map. Mol Breeding 24 293-303.

Spigler RB, Ashman T-L (2011). Sex ratio and subdioecy in Fragaria virginiana: the roles of plasticity and gene flow examined. New Phytol 190: 1058-1068.

Spigler RB, Lewers KS, Ashman TL (2011). Genetic architecture of sexual dimorphism in a subdioecious plant with a proto-sex chromosome. Evolution 65 1114-1126.

Spigler RB, Lewers KS, Johnson AL, Ashman T-L (2010). Comparative mapping reveals autosomal origin of sex chromosome in octoploid Fragaria virginiana. J Hered 101: S107-S117.

Spigler RB, Lewers KS, Main DS, Ashman T-L (2008). Genetic mapping of sex determination in a wild strawberry, Fragaria virginiana, reveals earliest form of sex chromosome. Heredity 101: 507-517.

Staudt G (1989). The species of Fragaria, their taxonomy and geographical distribution. Acta Hort 265: 23-34.

Staudt G (1999). Systematics and Geographic Distribution of the American Strawberry Species: Taxonomic Studies in the Genus Fragaria (Rosaceae: Potentilleae). University of California Press: Berkeley, CA, USA.
Sweigart A (2009). Sculpin hybrid zones: natural laboratories for the early stages of speciation. Mol Ecol 18: 2547-2548.

Van Ooijen JW (2004). MapQTL ${ }^{\circledR}$ 5, Software for the Mapping of Quantitative Trait Loci in Experimental Populations. Kyazma BV Wageningen: The Netherlands.

Van Ooijen JW (2006). JoinMap ${ }^{\circledR}$ 4, Software for the Calculation of Genetic Linkage Maps in Experimental Populations. Kyazma BV Wageningen: The Netherlands.

Veltsos P, Keller I, Nichols RA (2008). The inexorable spread of a newly arisen neo-Y chromosome. PLoS Genet 4: e1000082. EP.

Voorrips RE (2002). MapChart: software for the graphical of linkage maps and QTLs. J Hered 93: 77-78.

Wagner Jr WH (1970). Biosystematics and evolutionary noise. Taxon 19: 146-151.

Wallace LE, Culley TM, Weller SG, Sakai AK, Kuenzi A, Roy T et al. (2011). Asymmetrical gene flow in a hybrid zone of Hawaiian Schiedea (Caryophyllaceae) species with contrasting mating systems. PLOS ONE 6: e24845. EP.

Wu KK, Burnquist W, Sorrells ME, Tew TL, Moore PH, Tanksley SD (1992). The detection and estimation of linkage in polyploids using single-dose restriction fragments. Theor App/ Genet 83: 294-300.

Zluvova J, Lengerova M, Markova M, Hobza R, Nicolas M, Vyskot B et al. (2005). The interspecific hybrid Silene latifolia $\times$ S. viscosa reveals early events of sex chromosome evolution. Evol Dev 7: 327-336.

Supplementary Information accompanies the paper on Heredity website (http://www.nature.com/hdy) 